\title{
Centralized Care For Acute Pancreatitis Significantly Improves Outcomes
}

\author{
Szilárd Gódi ${ }^{1}$, Bálint Erőss ${ }^{1,2}$, Zsuzsanna Gyömbér ${ }^{3}$, Andrea Szentesi ${ }^{2,3}$, Nelli Farkas ${ }^{2,4}$, Andrea Párniczky ${ }^{2,5}$, Patrícia Sarlós $^{1,2}$,

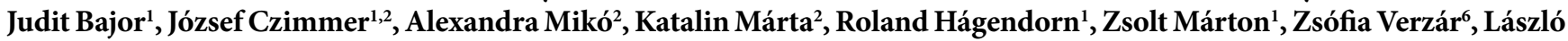 \\ Czakó $^{3}$, Zoltán Szepes ${ }^{3}$, Áron Vincze ${ }^{1,2^{*}}$, Péter Hegyi ${ }^{1,2,3,7^{*}}$ on behalf of the Hungarian Pancreatic Study Group
}

1) $1^{\text {st }}$ Department of Medicine, University of Pécs Medical School, 7624 Pécs,

2) Institute for Translational Medicine, University of Pécs Medical School, 7624 Pécs,

3) $1^{\text {st }}$ Department of Medicine, University of Szeged, 6720 Szeged,

4) Institute of Bioanalysis, University of Pécs Medical School, 7624 Pécs, Hungary 5) Department of Gastroenterology, Heim Pál Children's Hospital, 1098 Budapest,

6) Department of Emergency Medicine, University of Pécs School of Medicine, 7624 Pécs, 7) MTA-SZTE Momentum Gastroenterology Multidisciplinary Research Group, Hungary

Address for correspondence: Péter Hegyi Institute for Translational Medicine, University of Pécs Szigeti út 12., II. em., H-7624 Pécs, Hungary hegyi2009@gmail.com

Received: 15.02.2018 Accepted: 30.04.2018

* equally contributed. List of clinical collaborators contributors on page 156 .

\section{ABSTRACT}

Aims: In this observational study, we investigated whether specialized care improves outcomes for acute pancreatitis (AP).

Methods: Consecutive patients admitted to two university hospitals with AP were enrolled in this study between 1 January 2016 and 31 December 2016 (Center A: specialized center; Center B: general hospital). Data on demographic characteristics and AP etiology, severity, mortality and quality of care (enteral nutrition and antibiotic use) were extracted from the Hungarian Acute Pancreatitis Registry. An independent sample $t$-test, Mann-Whitney test, chi-squared test or Fisher's test were used for statistical analyses. Costs of care were calculated and compared in the two models of care.

Results: There were 355 patients enrolled, 195 patients in the specialized center (Center A) and 160 patients in the general hospital (Center $\mathrm{B})$. There was no difference in mean age $(57.02 \pm 17.16$ vs. $57.31 \pm 16.50 \mathrm{P}=0.872)$ and sex ratio ( $56 \%$ males vs. $57 \%$ males, $\mathrm{P}=0.837$ ) between centres, allowing a comparison without selection bias. Center A had lower mortality $(n=2,1.03 \%$ vs. $n=16,6.25 \%, p=0.007)$, more patients received enteral feeding $(n=179,91.8 \%$, vs. $n=36,22.5 \%, p<0.001)$ and fewer patients were treated with antibiotics $(n=85$, $43.6 \%$ vs. $n=123,76.9 \%, p=0.001)$. In Center A the median length of hospitalization was shorter (Me 6, IQR 5-9 vs. Me 8, IQR 6-11, $\mathrm{p}=0.02$ ) and the costs of care were by $25 \%$ lower.

Conclusion: Our data suggests that treatment of AP in specialized centers reduces mortality, length of hospitalization and thus might reduce the costs.

Key words: acute pancreatitis - costs - specialized center - outcome - mortality.

Abbreviations: ACG: American College of Gastroenterology; AP: Acute pancreatitis; ER: Emergency Unit; IAP/APA: International Association of Pancreatology and the American Pancreatic Association; ICU: Intensive Care Unit; LOH: Length of hospitalization; SD: Surgical Department; TIMD: Territorial Internal Medical Departments; TPC: Tertiary Pancreas Center.

\section{INTRODUCTION}

Medical care for acute diseases requiring hospitalization varies between countries and often within the same country. The same acute diseases are treated in hospitals with different profiles of expertise, different bed bases for specialties and different guidelines. There are two major pathways for admission with acute diseases.

One of the pathways is organized care in high-volume specialized centers for specific todiseases, where patients are directly admitted to a highly specialized ward, with a multidisciplinary team, strict adherence to guidelines and easy access to special procedures (Fig. 1A). There are examples of established specialized care models for the treatment of stroke and acute coronary syndrome $[1,2]$.

On the other pathway, patients are referred to general medical wards (internal medicine or surgery), and, depending on the progression of the disease, some patients are transferred to specialized wards. If there is further deterioration, transfer to an intensive care unit may be necessary. We define this as the general medical care model.

Outcomes for acute diseases can be significantly different depending on care, and there are examples of significantly improved outcomes for acute diseases treated in specialized centers. There is ample evidence that organized care for 
stroke [1] and acute myocardial infarction with ST elevation in specialized centers have saved lives and reduced the burden of these diseases [2]. Based on this evidence, national and international stroke [3] and cardiology [4] associations organized care in specialized centers, with specific recommendations in their guidelines.

Acute pancreatitis (AP) is the most common acute presentation in gastroenterology requiring hospital admission in the USA [5]. According to data obtained from the Hungarian National Health Insurance Fund, there is an estimated 5500 AP hospital admissions/year for Hungary's population of 10 million. There have been significant efforts to improve outcomes and to reduce the disease burden in AP as suboptimal care can result in progression to severe forms of the disease, higher morbidity and mortality. The Working Group of the International Association of Pancreatology and the American Pancreatic Association (IAP/APA) updated and published evidence-based guidelines for the treatment of AP [6] most recently in 2013, and the American College of Gastroenterology (ACG) also published their guidelines [7] the same year. The Hungarian Pancreatic Study Group translated both and synthesized them in the Hungarian guidelines [8] in 2015 .

The IAP/APA guidelines suggest intensive care for patients with severe AP and referral to a specialized center [6]. In defining a center specialized for AP, the guidelines specify the need for intensive care facilities for organ replacement therapy, continuous access to interventional radiology, endoscopic retrograde cholangiopancreatography (ERCP), endoscopic ultrasonography and surgical expertise in the treatment of necrotizing AP [6].

The ACG guidelines recommend risk stratification to identify patients who will need early transfer to an intensive care unit [7]. Referral to a specialized center in the case of severe AP is also recommended in the Hungarian guidelines [8]. However, there is no recommendation in these guidelines regarding whether all AP (mild and moderate) should be referred to centers with specialized care after diagnosis at the emergency unit. There is convincing evidence on improved outcomes for AP in high-volume centers (more than 118 admissions/year for AP), according to Singla et al. [9].

It is difficult to predict the severity of AP at the time of admission, and patients presenting with mild forms can develop fulminant AP within a few days. Current stratification systems for AP are unable to predict the course of the disease at the early stage unless the disease is severe at the time of admission. The revised Atlanta classification of AP severity is determined by clinical parameters recorded throughout the disease and provides disease severity in retrospect [10]. Therefore, it is not suitable for predicting the outcome.

The guidelines for the treatment of AP [7-9] recommend that the risk factors, the clinical prognostic factors and the response to the treatment should be monitored to predict the outcome. However, a reliable prediction system based on admission parameters is yet to be developed for the accurate prediction of the clinical course of and outcome for AP. Until now, no evidence has been published for or against the treatment of AP of all severities in specialized pancreatic centers.
Our aim was to investigate whether specialized care improves the outcomes for AP.

\section{METHODS}

The Hungarian Pancreatic Study Group was established in 2011 to improve care for pancreatic diseases. To date, the Hungarian Pancreatic Study Group has built up an international prospective registry for AP and organized five registered clinical trials to investigate AP under the acronyms PREPAST [11], APPLE [12], PINEAPPLE [13], GOULASH [14] and EASY [15].

\section{Study design}

In this observational cohort study, we analyzed and compared data from the prospectively collected AP Registry, specifically, outcomes, quality of care and costs for AP in two university hospitals with two different models for the management of AP.

Treatment Center A fulfilled the criteria for a specialized center for AP. Patients were admitted directly to the specialized ward from regional emergency departments. Center A admits patients from nine high-volume emergency units in the region. The specialized center has an integrated care pathway for patients requiring care in the high dependency unit or intensive care unit (Fig. 1A).

Treatment Center B admits patients with AP to general internal medicine wards from the emergency department, and patients are transferred to the specialized pancreatic ward, if there is deterioration. Treatment Center B transfers patients from the emergency department to one of the five general internal medicine wards or to a surgical ward, or if indicated by the patient's status, either to the tertiary pancreatic center or to the intensive care unit (Fig. 1B).

Both treatment centers (A and B) deal with high volumes, but their models of care for AP are different. Both institutions care for populations with nearly identical demographics.

\section{Limitation}

The study design and the differences between the two cohorts are potential sources of bias and limitation; therefore, both cohorts were carefully scrutinised through statistical analysis before comparing outcomes to ensure that they were comparable.

\section{Ethical approval}

The study was approved by the Scientific and Research Ethics Committee of the Medical Research Council (222541/2012/EKU) on 15 August 2012 and conforms to the 1975 Declaration of Helsinki ethical guidelines as reflected in a priori approval by the institution's human research committee. The patients signed the relevant consent forms.

\section{Statistical analyses}

The demographics and the etiology in both samples were compared. To analyze the differences in the distribution of severity, complications (local and systemic), mortality and management (enteral feeding and antibiotic use) between the centers, we used Pearson's chi-squared test or Fisher's exact 
$n=195$

Pancreatic Center $n=160$

General M. Center
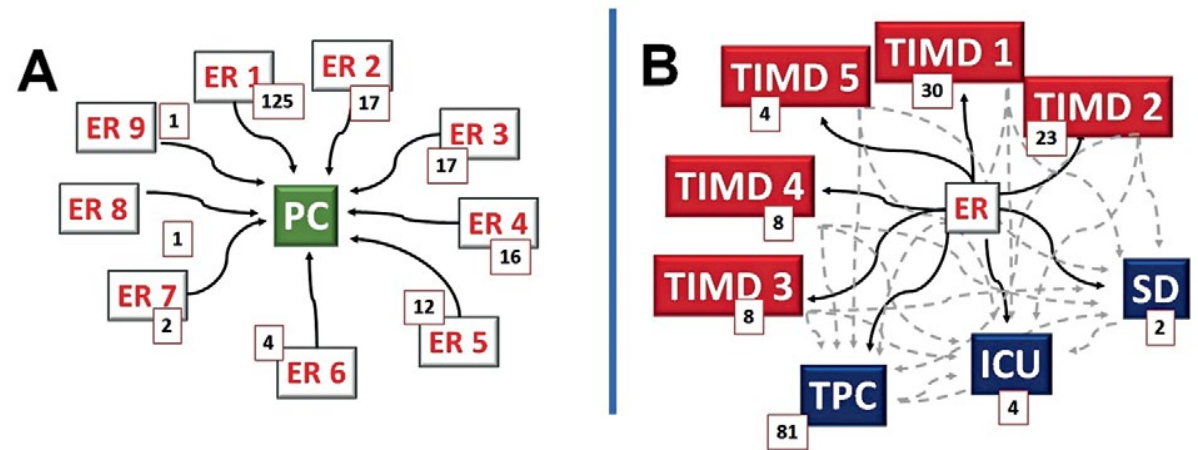

Fig. 1. A: Patient pathways in the specialized high-volume center from different Emergency Units (ER); B: Patient pathways in the step-up care pathway institution from Territorial Internal Medical Departments (TIMD), Intensive Care Units (ICU), the Tertiary Pancreas Center (TPC) and the Surgical Department (SD), number of cases (n).

test. We used the independent samples Student's $t$-test for age and the Mann-Whitney U test for comparison of hospital stay. $P$ values under 0.05 were considered statistically significant. Where the $\mathrm{p}$ value was less than 0.1 but higher than 0.05 , we only regarded the result as a tendency. All statistical analyses were performed by IBM SPSS Statistics v 24.0 (IBM, New York, NY, USA).

\section{RESULTS}

The best evidence in comparative medicine is always provided by the results from randomized clinical trials, as they ensure that there is no selection bias between cases and controls. However, in this observational trial, it was impossible to perform randomization, since there was only one model of care for AP in the two centers. In addition, these large centers are far away from each other; therefore, transfer of patients after randomization would not have been possible. Finally, and most importantly, randomization would have been unethical.
The two university centers are located in the same region of Europe with an ethnically homogeneous population, and $98 \%$ of the patients approached gave their consent at both centers.

\section{Demographic characteristics}

Treatment Center A with specialized care for AP admitted 195 patients, while treatment Center B treated 160 patients with AP in 2016 (Fig. 1A, B). A demographic analysis of the two cohorts showed no significant difference. Mean age was 57.02 $( \pm 17.16)$ in Center A and $57.31( \pm 16.50)$ in Center B $(p=0.872)$. The proportion of males was $56 \%$ in Center $A$ and $57 \%$ in Center $\mathrm{B}(\mathrm{p}=0.837)$. Age did not differ significantly in males or females between the two cohorts (male mean age in center A: 54.16 \pm 16.96 ; in Center B: $57.03 \pm 16.01, p=0.221$; female mean age in Center A: $60.71 \pm 16.80$; in Center B: $57.68 \pm 17.26, \mathrm{p}=0.276$ ).

Although it was not intentional, the cohorts were matched for age and sex (Fig. 2 A, B ).

The etiology of AP was similar in both cohorts. The major causes were biliary stones, alcohol, and idiopathic
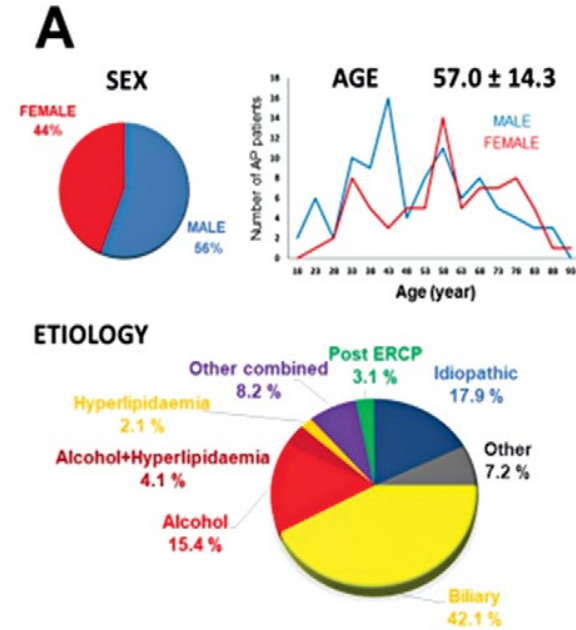

B
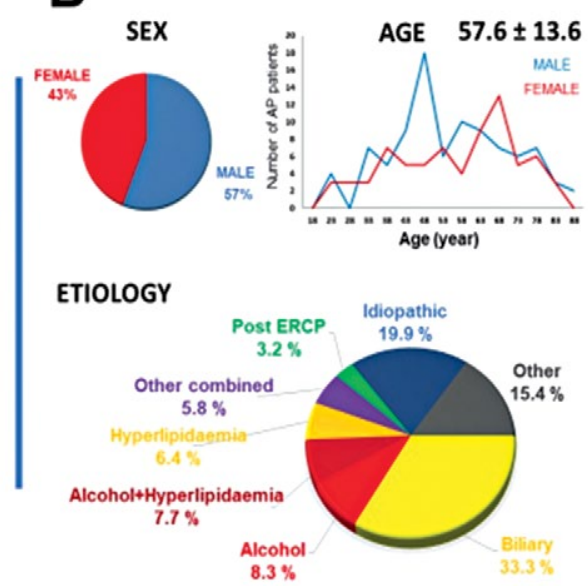

Fig. 2. A: The demographic characteristics and etiological factors of acute pancreatitis in the specialized high-volume center; B: The demographic characteristics and etiological factors of acute pancreatitis in the step-up care pathway institution. 
and hyperlipidemia, accounting for the vast majority in both centers (Fig. 2).

This similarity, along with the matched age and sex ratio, allowed us to compare the outcomes for AP in both cohorts and reduced the selection bias of this observational cohort study.

\section{Severity and mortality of AP}

The distribution of the worst severity of AP was different in Centers A and B (Fig. 3 A, B). Center B had 67\% more severe $\operatorname{AP}(n=19,11.9 \%)$ than Center A $(n=14,7.1 \%)$; however, this was not statistically different $(\mathrm{p}=0.310)$, likely due to the small sample sizes, 14 patients in Center A and 19 in Center B.

The mortality of all AP in Center B was six times higher $(n=16,6.25 \%)$ than in Center $A(n=2,1.03 \%)$, and this difference proved to be statistically significant, $\mathrm{p}=0.007$. Severe AP showed a threefold increase of mortality in Center $B(n=8$, $47.37 \%$ vs. $n=2,14.29 \%, p=0.067$ ). There were no deaths from mild AP in either cohort.

The average hospital stay was significantly shorter in Center A (Me: 6 (IQR: 5-9) days vs. Me: 8 (IQR: 6-11) days, p=0.02) (Fig. $3 \mathrm{~A}, \mathrm{~B}$ ). The subgroup analysis found shorter means of hospital stay for all grades of severity; however, it was only significant in mild AP, suggesting that mild cases of AP were discharged sooner from Center A.

\section{Complications of AP}

Our analysis found no differences between the local and systemic complications of AP between Centers A and B $(n=43$, $23.1 \%$ vs. $n=35,21.8 \%, p=0.872$, and $n=21,10.5 \%$ vs. $n=27$,
$16.9 \%, \mathrm{p}=0.177$, respectively). The detailed results are shown in Fig. 3C.

\section{Interventions and therapy}

There were no differences between Center A and B in the number of ERCP $(n=85,43.59 \%$ vs. $n=59,36.88 \%$, $\mathrm{p}=0.143)$, necrosectomy ( $\mathrm{n}=1,0.5 \%$ vs. $\mathrm{n}=2,1.25 \%, \mathrm{p}=0.793$ ) and radiological or endoscopic ultrasound-guided drainage procedures ( $\mathrm{n}=8,4.1 \%$ vs. $\mathrm{n}=2,1.2 \%, \mathrm{p}=0.118$ ).

We found no differences in the number of ERCPs for biliary pancreatitis, $n=69,83 \%$ ERCPs in $n=83$ patients in Center $\mathrm{A}$ and $\mathrm{n}=45,84.9 \%$ ERCPs in $\mathrm{n}=53$ patients in Center $\mathrm{B}(\mathrm{p}=0.817)$. The ERCPs in AP with biliary etiology were performed in $n=60$ mild, $n=4$ moderate, and $n=5$ severe AP cases in Center $A$ and $n=40, n=4$ and $n=1$ in Center B. None of these were significantly different.

\section{Quality of care and management}

We investigated whether management of patients and adherence to the guidelines differed in the two centers. The best and most reliable management markers which we could identify were enteral feeding and the use of antibiotics (Fig. $4 \mathrm{~A}, \mathrm{~B})$.

More patients had enteral feeding in Center $A$ than in Center $B(n=179,91.8 \%$ vs. $n=36,22.5 \%$, $p<0.001)$ (Fig. 4 A,B).

The use of antibiotics also differed; significantly fewer patients were treated with antibiotics in Center A in contrast to Center $B(n=85,43.6 \%$ vs. $n=123,76.9 \%, p<0.001)$ (Fig. 4 A, B). A detailed analysis of antibiotic use demonstrated a

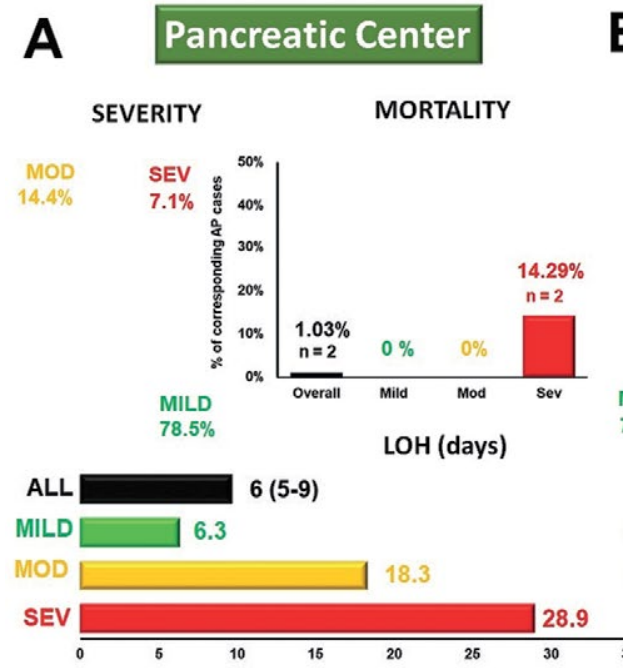

\section{B General M. Center}

\section{SEVERITY}

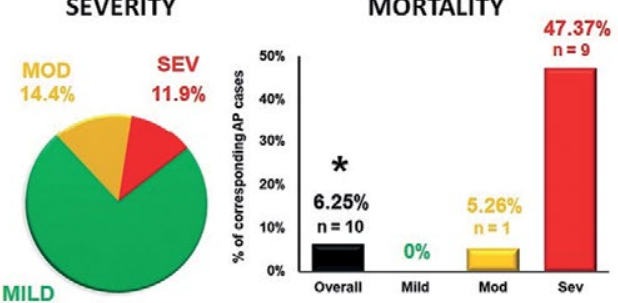

$73.8 \% \quad$ LOH (days)

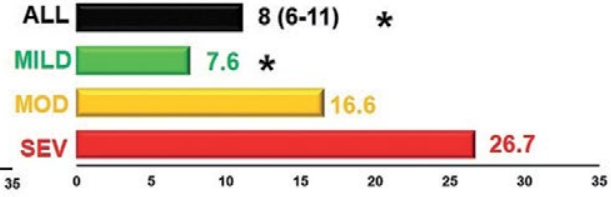

\section{C}

\begin{tabular}{|c|c|c|c|c|c|c|}
\hline & & \multicolumn{2}{|c|}{ CENTER A-Pancreatic center } & \multicolumn{2}{|c|}{ CENTER B-General M. Center } & $P$ value \\
\hline \multirow{3}{*}{ 气 } & no & 150 & $76.8 \%$ & 118 & $73.8 \%$ & 0.337 \\
\hline & local & 43 & $22.1 \%$ & 35 & $21.8 \%$ & 0.872 \\
\hline & systemic & 21 & $10.5 \%$ & 27 & $16.9 \%$ & 0.177 \\
\hline
\end{tabular}

Fig. 3. A: The distribution of disease severity, mortality and length of hospitalization (LOH) in the specialized high-volume center; B: The distribution of disease severity, mortality and length of hospitalization $(\mathrm{LOH})$ in the step-up care pathway institution; C: Complications in the two centers. ${ }^{*}$ significant difference; severe (SEV); moderate (MOD); number of cases (n). 


\section{A Pancreatic Center B General M. Center}

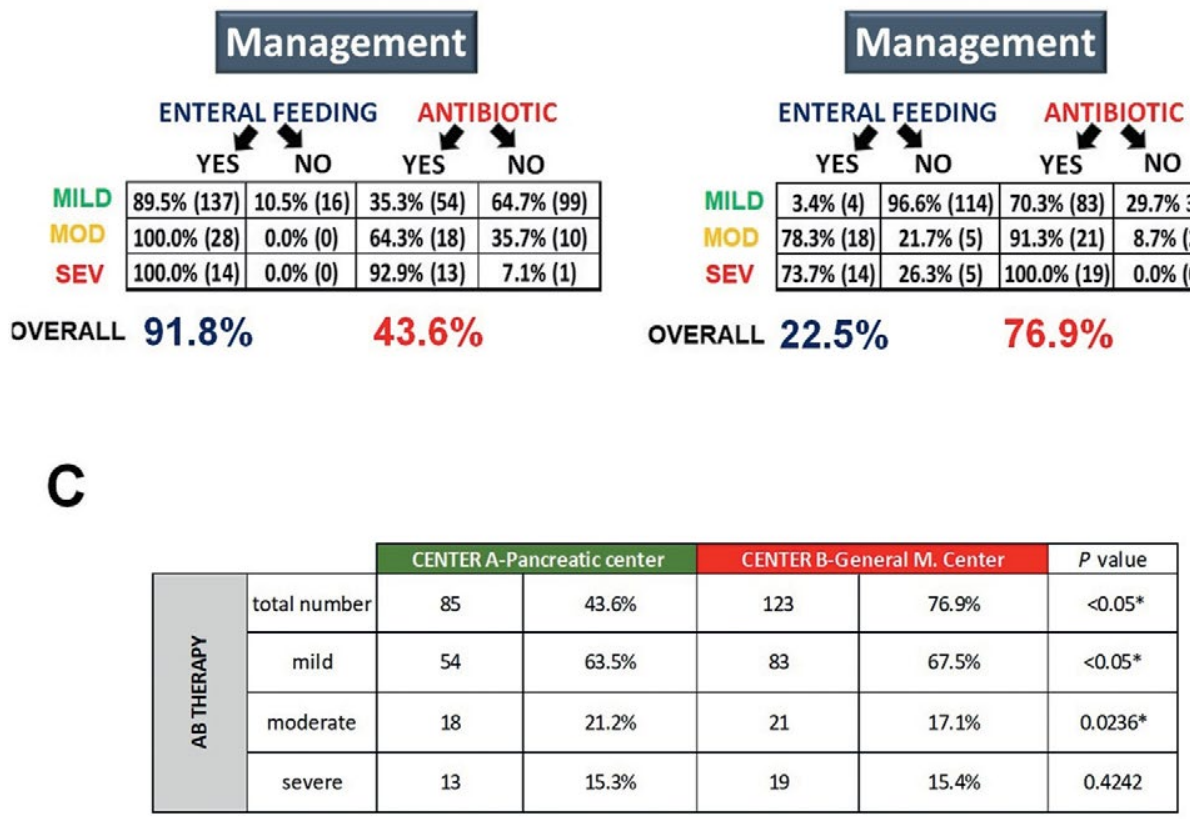

Fig. 4. A: Managing acute pancreatitis (with antibiotics and enteral nutrition) in the specialized high-volume center; B: Managing acute pancreatitis (with antibiotics and enteral nutrition) in the step-up care pathway institution; C: Antibiotic use in the two centers for different severities of acute pancreatitis. Severe (SEV); moderate (MOD).

significant difference between the two centers with regard to mild and moderate AP ( $\mathrm{n}=54,63.5 \%$, vs. $\mathrm{n}=83,67.5 \%, \mathrm{p}<0.05$ and $\mathrm{n}=18,21.2 \%$, vs. $\mathrm{n}=21,17.1 \%, \mathrm{p}=0.024)$. There was no difference in antibiotic use between the two centers with regard to severe AP $(n=13,15.3 \%$, vs. $n=19,15.4 \%, p=0.424)$. Results are shown on Fig. $4 \mathrm{C}$.

\section{Economic implications}

To understand the economic implications of the two different models of AP treatment, the average cost of management for 10 patients was calculated in both groups. The calculated average costs are based on the costs of medication, disposables, procedures and investigations. However, our calculation was limited by costs that could not be estimated, such as the costs of staff and hospital stay (Fig. 5).

The average daily costs in both centers in subgroups of mild, moderate and severe AP are indicated with or without antibiotic treatment. Based on this estimate, the cost of treatment for AP is $25 \%$ lower in the specialized care model than in the general medical model.

\section{DISCUSSION}

We hypothesized that specialized care for AP in highvolume centers is beneficial and measurable in both outcomes and costs. We analyzed comparable cohorts in both centers, without significant differences in their demographics. Both centers are tertiary referral institutions and university hospitals with the same level of medical expertise and skills. Funding for health care services in both centers is identical.

\section{Average Daily Costs of AP Therapy}

\begin{tabular}{|l|c|c|}
\cline { 2 - 3 } \multicolumn{1}{c|}{ Values in $€$} & $\mathrm{AB}+$ & $\mathrm{AB}-$ \\
\hline MILD & $\mathbf{7 6}$ & $\mathbf{7 1}$ \\
\hline MODERATE & 114 & 106 \\
\hline SEVERE & 151 & 142 \\
\hline
\end{tabular}

Average Costs of AP Therapy per Patient

Pancreatic Center

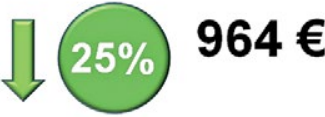

Fig. 5. A: Costs of managing acute pancreatitis (AP) in the specialized high-volume center; B: Costs of managing acute pancreatitis (AP) in the general medical center. This estimate does not include the costs of staff and hospital bed.

The only significant difference in terms of AP is their model of management of AP.

We believe that these prospectively collected data from the two cohorts are nearly as comparable as data from two branches of a randomized controlled trial, and we note that the latter would have been impossible and unethical to organize. 
There are multiple reasons we were able to show significantly improved outcomes, management and hospital stay in the specialized center.

In our analysis, we found that lower mortality and shorter hospital stay were associated with significant differences in the practices between the two centers. The specialized center with better outcomes used significantly more often enteral feeding and fewer antibiotics. However, there was no difference in the number of endoscopic or radiological procedures.

Center B followed the guidelines of enteral tube feeding more rigorously than Center A and limited the use of enteral tube feeding to manage patients with severe pancreatitis and predicted severe AP. We acknowledge that Center A used more enteral feeding to treat AP than strict adherence to the guidelines [8] would have suggested. There are many studies with evidence for early feeding in AP, but they compared enteral nutrition or enteral tube feeding vs. nil per os management. We note that there is a lack of clinical trials providing evidence and information on early oral vs. enteral tube feeding.

Petrov et al. [16] reported the benefits of enteral tube feeding in a randomized control trial compared to the nil per os approach and concluded that it leads to less oral food intolerance. Furthermore, our recent meta-analysis by Marta et al. [17] confirmed this. In addition, as a leading pancreatic clinical research unit, Center A is conducting a long-term randomized clinical trial investigating the benefits of early high-energy enteral tube nutrition in AP [14]. Prediction of severe AP is difficult in the early phase of the disease (24-48 hours). Enteral tube feeding may have the potential to prevent severe AP, and this is one of the foci of our research. Patients in Center A started oral feeding once their abdominal pain resolved, and enteral tube feeding often lasted less than one or two days in cases of mild and moderate AP.

Moraes et al. [18] and Larino-Noia et al. [19] showed that early oral re-feeding was safe and well tolerated, but neither study compared it to enteral tube feeding.

Finally, yet importantly, none of the guidelines precludes the option of enteral tube feeding in mild or moderate AP.

In Center A, with high-volume specialized care, patients are reviewed within 24 hours by gastroenterologists with expertise in AP, and patients are looked after by that same team throughout their hospital stay. Therefore, their continuity of care is optimal.

In Center B, patients are admitted to a general internal medicine unit, often without expertise in gastroenterology. We believe that this profound difference results in a significant delay in decision making in the treatment of AP, translated to poorer mortality. Patients transferred between medical teams often receive more fragmented treatment, and this approach increases the possibility of further delays and risks of complications as well.

Other possible factors are suboptimal knowledge and adherence to the AP guidelines among physicians without expertise and low case numbersof AP.

Based on these results, organized specialized care for AP in Hungary could shorten the hospital stay by 1,100 days ( 2 days/patient) and could save 275 lives (5\% more) in a single year. Specialized care could reduce the costs of medications, disposables, procedures and investigations by $25 \%$.

\section{CONCLUSION}

Managing AP in a high-volume center can potentially decrease disease severity, reduce the need for medications, improve mortality, shorten hospital stay and reduce costs of care. Therefore, further in-depth analysis would be warranted to establish whether AP should be managed in high-volume specialized centers.

Conflicts of interest: No conflicts to declare.

Authors' contributions: G.S. and H.P. formulated the research questions and designed the study. P.A., G.Z., S.A., G.S. and H.P. interpreted the data. F.N. performed the statistical analysis. G.S., M.A., E.B. and H.P. wrote the manuscript. The other authors contributed to the implementation of the study and the data acquisition. All authors read and approved the final manuscript.

ORCHID numbers of the authors (See Supplementary Material)

\section{Clinical collaborators:}

Dezső Kelemen, Róbert Papp (Department of Surgery, University of Pécs School of Medicine, Pécs, Hungary), Nándor Faluhelyi, Krisztián Molnár, Péter Farkas (Department of Radiology, University of Pécs School of Medicine, Pécs, Hungary), Tamás Takács ( $1^{\text {st }}$ Department of Medicine, University of Szeged, Szeged, Hungary), József Fenyő (Department of Emergency Medicine, St. Rókus Hospital, Baja, Hungary), Előd Papp (Department of Internal Medicine, St. Lukács Hospital, Dombóvár, Hungary), István Késői (Department of Internal Medicine, Komló General Hospital, Komló, Hungary), Balázs Marton (Department of Emergency Medicine, Mohács General Hospital, Mohács, Hungary), Csaba Csizmadia (Department of Internal Medicine, Mohács General Hospital, Mohács, Hungary), János Simon (Department of Emergency Medicine, Balassa János Tolna County Hospital, Szekszárd, Hungary), Ágnes Salamon (Department of Internal Medicine, Balassa János Tolna County Hospital, Szekszárd, Hungary), Éva Szabó (Department of Internal Medicine, Szigetvár General Hospital, Szigetvár, Hungary)

Acknowledgement: The study was supported by a Project Grant (KH125678 to $\mathrm{PH}$ ), an Economic Development and Innovation Operative Program Grant (GINOP 2.3.2-15-2016-00048 to PH) and a Human Resources Development Operational Program Grant (EFOP-3.6.2-16-2017-00006 to PH) from the National Research, Development and Innovation Office as well as by a Momentum Grant from the Hungarian Academy of Sciences (LP2014-10/2014 to PH) and the UNKP-17-3-I. New National Excellence Program, Ministry of Human Capacities (PTE/46539/2017 to KM).

Supplementary material: To access the supplementary material visit the online version of the J Gastrointestin Liver Dis at http://dx.doi. org/10.15403/jgld.2014.1121.272.pan

\section{REFERENCES}

1. Stroke Unit Trialists' Collaboration. Organised inpatient (stroke unit) care for stroke. Cochrane Database Syst Rev 2013;(9):CD000197. doi:10.1002/14651858.CD000197.pub3 
2. Keller T, Post F, Tzikas S, et al. Improved outcome in acute coronary syndrome by establishing a chest pain unit. Clin Res Cardiol 2010;99:149-155. doi:10.1007/s00392-009-0099-9

3. Jauch EC, Saver JL, Adams HP Jr, et al. Guidelines for the early management of patients with acute ischemic stroke: a guideline for healthcare professionals from the American Heart Association/American Stroke Association. Stroke 2013;44:870-947. doi:10.1161/STR.0b013e318284056a

4. Ibanez B, James S, Agewall S, et al. 2017 ESC Guidelines for the management of acute myocardial infarction in patients presenting with ST-segment elevation: The Task Force for the management of acute myocardial infarction in patients presenting with ST-segment elevation of the European Society of Cardiology (ESC). Eur Heart 2018;39:119-177. doi:10.1093/eurheartj/ehx393

5. Peery AF, Dellon ES, Lund J, et al. Burden of gastrointestinal disease in the United States: 2012 update. Gastroenterology 2012;143:1179-1187. e1-3. doi:10.1053/j.gastro.2012.08.002

6. Working Group IAP/APA Acute Pancreatitis Guidelines. IAP/APA evidence-based guidelines for the management of acute pancreatitis. Pancreatology 2013;13(4 Suppl 2):e1-15. doi:10.1016/j.pan.2013.07.063

7. Tenner S, Baillie J, DeWitt J, Vege SS. American College of Gastroenterology guideline: management of acute pancreatitis. Am J Gastroenterol 2013;108:1400-1415. doi:10.1038/ajg.2013.218

8. Hritz I, Czako L, Dubravcsik Z, et al. Acute pancreatitis. Evidence-based practice guidelines, prepared by the Hungarian Pancreatic Study Group Orv Hetil 2015;156:244-261. doi:10.1556/oh.2015.30059

9. Singla A, Simons J, Li Y, et al. Admission volume determines outcome for patients with acute pancreatitis. Gastroenterology 2009;137:19952001. doi:10.1053/j.gastro.2009.08.056

10. Banks PA, Bollen TL, Dervenis C, et al. Classification of acute pancreatitis--2012: revision of the Atlanta classification and definitions by international consensus. Gut 2013;62:102-111. doi:10.1136/ gutjnl-2012-302779

11. Dubravcsik Z, Madacsy L, Gyokeres T, et al. Preventive pancreatic stents in the management of acute biliary pancreatitis (PREPAST trial): pre-study protocol for a multicenter, prospective, randomized, interventional, controlled trial. Pancreatology 2015;15:115-123. doi:10.1016/j.pan.2015.02.007

12. Parniczky A, Mosztbacher D, Zsoldos F, Toth A, Lasztity N, Hegyi P. Analysis of Pediatric Pancreatitis (APPLE Trial): Pre-Study Protocol of a Multinational Prospective Clinical Trial. Digestion 2016;93:105-110. doi:10.1159/000441353

13. Zsoldos F, Parniczky A, Mosztbacher D, Toth A, Lasztity N, Hegyi P. Pain in the Early Phase of Pediatric Pancreatitis (PINEAPPLE Trial): PreStudy Protocol of a Multinational Prospective Clinical Trial. Digestion 2016;93:121-126. doi:10.1159/000441352

14. Marta K, Szabo AN, Pecsi D, et al. High versus low energy administration in the early phase of acute pancreatitis (GOULASH trial): protocol of a multicentre randomised double-blind clinical trial. BMJ Open 2017;7:e015874. doi:10.1136/bmjopen-2017-015874

15. Hritz I, Hegyi P. Early Achievable Severity (EASY) index for simple and accurate expedite risk stratification in acute pancreatitis. J Gastrointestin Liver Dis 2015;24:177-182. doi:10.15403/jgld.2014.1121.242.easy

16. Petrov MS, McIlroy K, Grayson L, Phillips AR, Windsor JA. Early nasogastric tube feeding versus nil per os in mild to moderate acute pancreatitis: a randomized controlled trial. Clin Nutr 2013;32:697-703. doi:10.1016/j.clnu.2012.12.011

17. Márta K, Farkas N, Szabó I, et al. Meta-Analysis of Early Nutrition: The Benefits of Enteral Feeding Compared to a Nil Per Os Diet Not Only in Severe, but Also in Mild and Moderate Acute Pancreatitis. Int J Mol Sci 2016;17:1691. doi:10.3390/ijms17101691

18. Moraes JM, Felga GE, Chebli LA, et al. A full solid diet as the initial meal in mild acute pancreatitis is safe and result in a shorter length of hospitalization: results from a prospective, randomized, controlled, double-blind clinical trial. J Clin Gastroenterol 2010;44:517-522. doi:10.1097/MCG.0b013e3181c986b3

19. Lariño-Noia J, Lindkvist B, Iglesias-García J, Seijo-Ríos S, Iglesias-Canle J, Domínguez-Muñoz JE. Early and/or immediately full caloric diet versus standard refeeding in mild acute pancreatitis: a randomized open-label trial. Pancreatology 2014;14:167-173. doi:10.1016/j. pan.2014.02.008 


\section{ORCID members}

Szilárd Gódi (0000-0003-2395-4336), Bálint Erőss (0000-0003-3658-8427), Zsuzsanna Gyömbér (0000-0001-6978-7786), Andrea Szentesi (0000-0003-2097-6927), Nelli Farkas (0000-0002-5349-6527), Andrea Párniczky (0000-0003-3466-4780), Áron Vincze (00000003-2217-7686 ), Patrícia Sarlós (0000-0002-5086-9455), Judit Bajor (0000-0002-39414871), József Czimmer (0000-0001-7831-3523), Alexandra Mikó (0000-0002-53224425), Katalin Márta (0000-0002-2213-4865), Roland Hágendorn (0000-0002-99842309), Zsolt Márton (0000-0003-3314-4513), Dezső Kelemen (0000-0003-0093-6813), Róbert Papp (0000-0001-7699-1806), Nándor Faluhelyi (0000-0002-3786-9215), Krisztián Molnár (0000-0002-2347-460X), Péter Farkas (0000-0001-5593-1898), Zsófia Verzár (0000-0001-5323-0748), László Czakó (0000-0002-6331-0802), Tamás Takács (0000-0003-3321-8690), Zoltán Szepes (0000-0002-9466-8719), József Fenyő (0000-00033963-6578), Előd Papp (0000-0003-4536-0117), István Késői (0000-0002-7112-628X), Balázs Marton (0000-0002-0635-9474), Csaba Csizmadia (0000-0002-5334-0063), János Simon (0000-0002-8324-4718), Ágnes Salamon (0000-0002-7198-3708), Éva Szabó (0000-0002-2250-1979) and Péter Hegyi (0000-0003-0399-7259) 